\title{
BMJ Open Healthcare utilisation trajectories in patients dying from chronic obstructive pulmonary disease, heart failure or cancer: a nationwide register-based cohort study
}

\author{
Anne Høy Seemann Vestergaard (D) ,1,2 Christian Fynbo Christiansen (D) ,,2 \\ Mette Asbjoern Neergaard, ${ }^{3}$ Jan Brink Valentin (iD , ${ }^{4,5}$ Søren Paaske Johnsen ${ }^{4,5}$
}

To cite: Vestergaard AHS, Christiansen CF, Neergaard MA, et al. Healthcare utilisation trajectories in patients dying from chronic obstructive pulmonary disease, heart failure or cancer: a nationwide registerbased cohort study. BMJ Open 2021;11:e049661. doi:10.1136/ bmjopen-2021-049661

- Prepublication history for this paper is available online. To view these files, please visit the journal online (http://dx.doi. org/10.1136/bmjopen-2021049661).

Received 29 January 2021 Accepted 21 October 2021

Check for updates

(C) Author(s) (or their employer(s)) 2021. Re-use permitted under CC BY-NC. No commercial re-use. See rights and permissions. Published by BMJ.

For numbered affiliations see end of article.

Correspondence to

Dr Anne Høy Seemann

Vestergaard; ahsv@clin.au.dk

\section{ABSTRACT}

Objectives To investigate illness trajectories as reflected by healthcare utilisation, including hospital and intensive care unit admissions, consultations in general practice and home care provision, before death comparing people dying from chronic obstructive pulmonary disease (COPD), heart failure and cancer.

Design Nationwide register-based cohort study. Setting Data on all hospital admissions, including intensive care unit admissions, consultations in general practice and home care provision were obtained from nationwide Danish registries.

Participants All adult decedents in Denmark dying from COPD, heart failure or cancer between 2006 and 2016.

Outcome measures For each day within 5 years before death, we computed a daily prevalence proportion (PP) of being admitted to hospital or consulting a general practitioner. For each day within 6 months before death, we computed PPs of being admitted to intensive care or receiving home care. The PPs were plotted and compared by regression analyses adjusting for age, gender,

comorbidity level, marital/cohabitation status, municipality and income level.

Results Among 174086 patients dying from COPD $(n=22648)$, heart failure $(n=11498)$ or cancer $(n=139$ 940), the PPs of being admitted to hospital or consulting a general practitioner showed similar steady progression and steep increase in the last year of life for all patient populations. The PP of being admitted to intensive care showed modest increase during the last 6 months of life, accelerating in the last month, for all patient populations. For patients with COPD and heart failure, the PP of receiving home care remained stable during the last 6 months of life but increased steadily for patients with cancer.

Conclusion We found limited differences in healthcare resource utilisation at the end of life for people with COPD, heart failure or cancer, indicating comparable illness trajectories.

This supports the need to reconsider efforts in achieving equal access to palliative care interventions, which is still mainly offered to patients with cancer.
Strengths and limitations of this study

- The main strengths included the nationwide population-based design in a uniformly organised healthcare system and accurate linkage between national medical registries.

- The study included routinely and prospectively collected data from Danish registries, which in general are considered to have high validity.

- Analyses were based on the underlying cause of death of well-defined chronic diseases in order to minimise misclassification between underlying and immediate cause of death.

- The study was based on the assumption of healthcare resource utilisation being a marker of illness progression and thereby functional impairment and symptom burden at the end of life, but healthcare utilisation may not accurately reflect performance status or palliative care needs.

\section{INTRODUCTION}

Despite growing body of evidence of efficacious palliative care for patients with non-cancer diseases, ${ }^{1-7}$ palliative care interventions are still mainly offered to patients with cancer. ${ }^{8}$ The reason for this inequality is probably multifaceted, but possible reasons have been suggested to be lacking recognition of non-cancer diseases as life-threatening and difficulties in predicting the functional decline of the illness trajectory in these patients. ${ }^{1011}$ Thus, inspection of detailed health data on the trajectories of non-cancer diseases compared with cancer trajectories may provide insights that will benefit future patients.

The trajectories of decline in terms of physical function are not directly registered in national healthcare registries, but healthcare resource utilisation may be a useful marker of functional impairment and symptom burden of the underlying disease ${ }^{12}$ and thereby 
provide a measure of severity and progression of the disease. ${ }^{1314}$

To our knowledge, no previous study has investigated the illness trajectories of non-cancer diseases in a large nationwide study and compared this with the trajectories of different cancer types in the years before death. We therefore aimed to examine illness trajectories of people dying from chronic obstructive pulmonary disease (COPD), heart failure and cancer by estimating the prevalence proportion (PP) of place of care on any given day in their last years of life including all-cause hospital admissions and consultations in general practice within 5 years before death and intensive care unit admissions and home care provision within 6 months before death.

\section{METHODS}

\section{Study design and setting}

A nationwide register-based study was conducted among all adult decedents who died from COPD, heart failure or cancer in Denmark between 1 January 2006 and 31 December 2016.

Using the unique civil registration number assigned to all Danish residents at birth or immigration, individuallevel records were linked across databases. ${ }^{15}$ Since 1968, the civil registration number has been used in public Danish registries, permitting accurate linkage between all national registries. ${ }^{15}$

The healthcare system in Denmark is public and provides tax-supported healthcare to all residents who have equal access to healthcare, including access to public hospitals, hospices, general practitioners, home care and specialists in palliative care. ${ }^{16}$

\section{Patient and public involvement}

Neither patients nor public members were involved in this research.

\section{Decedents}

Using the Danish Registry of Causes of Death, we identified all decedents in Denmark registered with COPD, heart failure or cancer (including all types of cancer) as the underlying cause of death. We only included decedents 18 years or older who had been residents in Denmark for at least 5 years prior to death in order to have complete data on the use of healthcare services at the end of life.

For the study of hospital admissions and consultations in general practice, we included decedents who died between 1 January 2006 and 31 December 2016, whereas for the study of intensive care unit admissions and home care provision, we identified decedents who died between 1 June 2011 and 31 December 2016 in order to comply with availability of data from the different registries (see description of the registries below).

The Danish Registry of Causes of Death is a nationwide registry with data collection from death certificates filled for every Danish decedent since 1970 with a completeness of approximately $97 \% .{ }^{17}$ The registry holds information on date of death and cause of death, coded according to the Danish version of International Classification of Diseases (10th edition, ICD-10). ${ }^{18}$

To ensure independence between the patient populations dying from COPD, heart failure or cancer, respectively, we excluded people dying from of one of the three conditions while also having any history of one or both of the other conditions recorded in the Danish National Patient Registry.

The Danish National Patient Registry is a nationwide registry containing records on hospital admissions since 1977. It is mandatory for all Danish hospitals to register information on hospital admissions, including dates of all admissions and discharges, patients' discharge diagnoses, surgical procedures and patients' residence. ${ }^{19}$

We also used the Danish National Registry of Patients to retrieve data on age at death, gender, residential municipality and region and comorbidity. We computed the Charlson Comorbidity Index based on all diagnoses in relation to hospitalisation and outpatient visits for each patient within 10 years leading up to death. The weights of 19 selected conditions were summed to a comorbidity score excluding the disease causing death, for example, for decedents dying from COPD, COPD was excluded in the calculation of their comorbidity score. ${ }^{20} 21$

The Danish Civil Registration System was used to obtain data regarding marital/cohabitation status for all patients, that is, living with a partner or not. ${ }^{22}$

Data on decedents' household income were obtained from Statistics Denmark, the central authority on Danish statistics with data on the Danish society. ${ }^{2324}$ Mean annual household income during the 5 year before the year of death was grouped into low income level (less than $50 \%$ of the national median), middle income level (50\%-100\% of the national median) and high income level (above the national median). National medians of household income in the year of death were obtained from Statistics Denmark. ${ }^{25}$

\section{Hospital admissions}

Data on all hospital admissions within 5 years before death were obtained using the Danish National Registry of Patients.

\section{Consultations in general practice}

Using the National Health Insurance Service Registry, we identified all in-office consultations in general practice as well as home visits provided by general practitioners for all included decedents within 5 years before death.

The National Health Insurance Service Registry has been effective since 1990 and is based on information on payments reimbursed by the National Health Insurance, wherefore reporting of data is assumed to be reliable. The National Health Insurance Service Registry covers all residents in Denmark and holds information on a range of primary care health services, including consultations with general practitioners to whom $98 \%$ of the residents are assigned. ${ }^{26}$ 


\section{Intensive care unit admissions}

Data on intensive care unit admissions within 6 months before death were obtained from the Danish National Registry of Patients using the algorithm developed by the Danish Intensive Care Database, a nationwide clinical quality database. ${ }^{27}$ The coding of intensive care admissions in the Danish National Registry of Patients is reported to have a positive predictive value of $98.7 \% .^{28}$ The estimated completeness for recordings on the exact date and time of intensive care unit discharge ranged from $78.7 \%$ in 2011 to $95.1 \%$ in $2015,{ }^{27}$ wherefore we decided only to include decedents dying between 1 June 2011 and 31 December 2016 and data on intensive care unit admissions within 6 months before death.

\section{Home care provision}

Using home care documentation provided by the Statistics Denmark, we obtained data on home care provision within 6 months before. The home care provision indicator was collected from 2011 and onwards, but data have not been entirely complete since then. Therefore, we only chose to include patients dying between 1 June 2011 and 31 December 2016 if the recordings on home care provision data in the municipality, they resided in, had been stated valid by the Statistics Denmark within the last 6 months leading up to death. ${ }^{29}$

The Statistics Denmark provides a collection of registry data containing information on each Danish resident and the Danish society, and since 2006, information have been collected directly from the municipalities' care systems into the Statistics Denmark. ${ }^{30}$

In Denmark, the municipalities are responsible for home care services and home nursing. ${ }^{16}$ There are two different categories of home care in Denmark, practical help and personal care, and those are provided free of charge for every Danish resident. In the current study, we only obtained information on personal care, since this offers the best measure of actual inabilities and progression of illness.

\section{Statistical methods}

Descriptive statistics were used to describe the cohort according to the underlying cause of death.

Daily PPs of being admitted to hospital, consulting a general practitioner, being admitted to intensive care units or receiving home care were computed for the three patient populations. This was calculated as the daily number of decedents admitted to hospital or consulting a general practitioner, respectively, divided by the number of patients in the study population on the specific date within 5 years before death. The daily PPs of patients admitted to an intensive care unit or receiving home care were calculated as the number of decedents admitted to an intensive care unit or receiving home care divided on the date by the number of decedents in the study population on the specific date within 6 months before death. For the analyses on home care provision, we excluded decedents who lived in municipalities in which recordings of home care provision data had not been stated valid in the 6 months leading up to death. We did this after making analyses showing that the number of decedents excluded were equally distributed in number, gender, age at death, cohabiting status and comorbidity between the patient populations.

The daily PPs of patients being admitted to hospital, consulting a general practitioner, being admitted to intensive care or receiving home care were graphed according to underlying cause of death for the three patient populations, and tabulated at 5 years, 1 year, 6 months and 30 days before death, respectively (only at 6 months and 30 days before death for the study of intensive care unit admissions and home care provision).

Correspondingly, daily PPs were computed and graphed according to major cancer types (lung cancer, breast cancer, prostate cancer, colon cancer, pancreatic cancer and haematological cancer).$^{31}$

We estimated daily prevalence proportion ratios (PPR) of patients being admitted to hospital, consulting a general practitioner, being admitted to intensive care or receiving home care for people who died of COPD or heart failure compared with patients who died of cancer. The daily PPR were adjusted for age at death, gender, comorbidity level, cohabitation status, municipality and income level using a Poisson regression model with robust variance estimator.

The adjusted daily PPR of being admitted to hospital, admitted to an intensive care unit, consulting a general practitioner or receiving home care were graphed, comparing patients dying from COPD and heart failure with patients with cancer.

The statistical analyses were performed using STATA V.16.1 (StataCorp. 2019. Stata Statistical Software: Release 16. College Station, Texas, USA: StataCorp LP) on a secure remote server at Statistics Denmark.

\section{RESULTS}

We identified 222693 patients above 18 years of age who died from COPD $(n=36626)$, heart failure $(n=16293)$ or cancer $(n=169774)$ in Denmark from 1 January 2006 to 31 December 2016. Patients dying from one of the three conditions while also having a history of one or both of the others were excluded (COPD: $\mathrm{n}=13978$; heart failure: $\mathrm{n}=4795$; cancer: $\mathrm{n}=29834$ ), leaving 174086 decedents for further analysis. Thus, for the study of hospital admissions and consultations in general practice, we identified 174086 patients who died of COPD $(n=22648)$, heart failure $(n=11498)$ or cancer $(n=139940)$, in Denmark from 1 January 2006 to 31 December 2016 (table 1).

For the study of intensive care in the period 1 June 2011 to 31 December 2016, we identified 87273 patients who died of COPD $(n=11731)$, heart failure $(n=5255)$ or cancer $(n=70287)$. From this patient population, we extracted 61286 patients (COPD: $n=8313$; heart 
Table 1 Characteristics of patients who died of cancer, chronic obstructive pulmonary disease (COPD) or heart failure in Denmark between 2006 and 2016

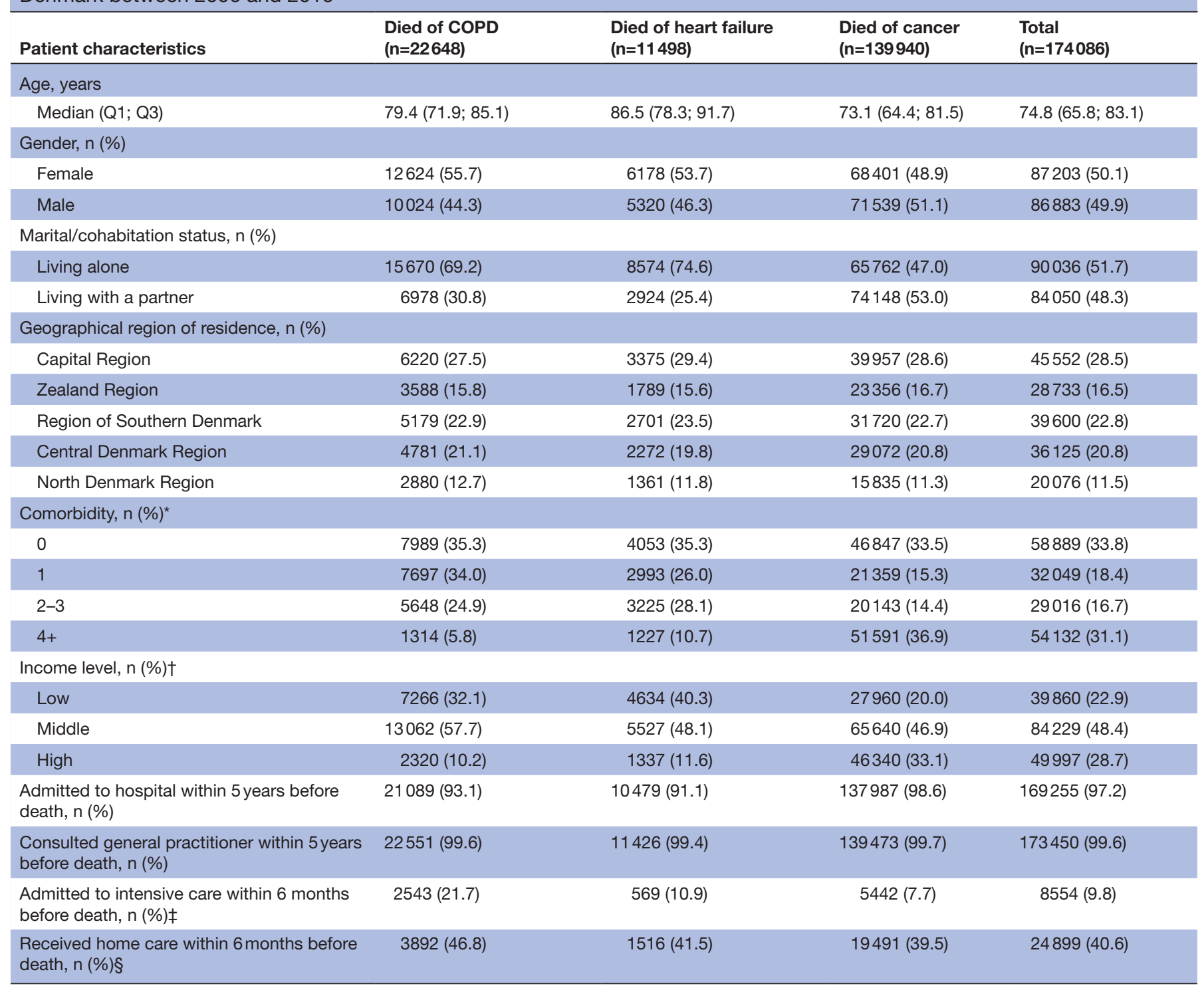

${ }^{*}$ Calculated according to Charlson Comorbidity, excluding underlying cause of death

†Income: low: less than $50 \%$ of national median; middle: above $50 \%$ of national median, but below national median; high: above national median.

†Patients who died in Denmark from 1 June 2011 to 31 December 2016 (COPD: $n=11731$; heart failure: $n=5255$; cancer: $n=70287$ ).

§Patients living in municipalities with acceptable level of data recording in the 6 months leading up to death and died in Denmark from 1 June 2011 to 31 December 2016 (COPD: $n=8313$; heart failure: $n=3652$; cancer: $n=49321$ ).

failure: $\mathrm{n}=3652$; cancer: $\mathrm{n}=49$ 321) living in municipalities in which recordings of home care provision had been stated valid in the 6 months leading up to death (table 1).

\section{Hospital admissions}

Among the decedents, $97.2 \%$ were admitted to hospital at least once within the last 5 years of life. Figure 1 shows that the daily PP of patients admitted to hospital steadily progressed for all patient populations until the last year before death, in which it increased steeply. Hence, at 5 years before death, the daily PP of patients admitted to hospital was $1.2 \%$ (95\% CI $1.1 \%$ to $1.4 \%$ ) for patients with COPD, $1.2 \%$ (95\% CI $1.0 \%$ to $1.5 \%$ ) for patients with heart failure and $0.7 \%$ (95\% CI $0.6 \%$ to $0.7 \%$ ) for patients with cancer, whereas the daily PP of patients admitted to hospital at day 30 before death was $11.0 \%$ (95\% CI $10.6 \%$ to $11.5 \%$ ) for patients with COPD, $11.2 \%$ (95\% CI $10.6 \%$ to $11.8 \%$ ) for patients with heart failure and $22.0 \%$ (95\% CI $21.7 \%$ to $22.2 \%$ ) for patients with cancer (table 2).

The adjusted daily PPR for COPD and heart failure patients when compared with patients with cancer decreased gradually during the 5 year period leading up to death (figure 2).

Stratifying on cancer type revealed no differences in the course of daily PP between the non-cancer diseases, COPD and heart failure, and the specific types of cancer within 5 years before death (figure 3 ). 

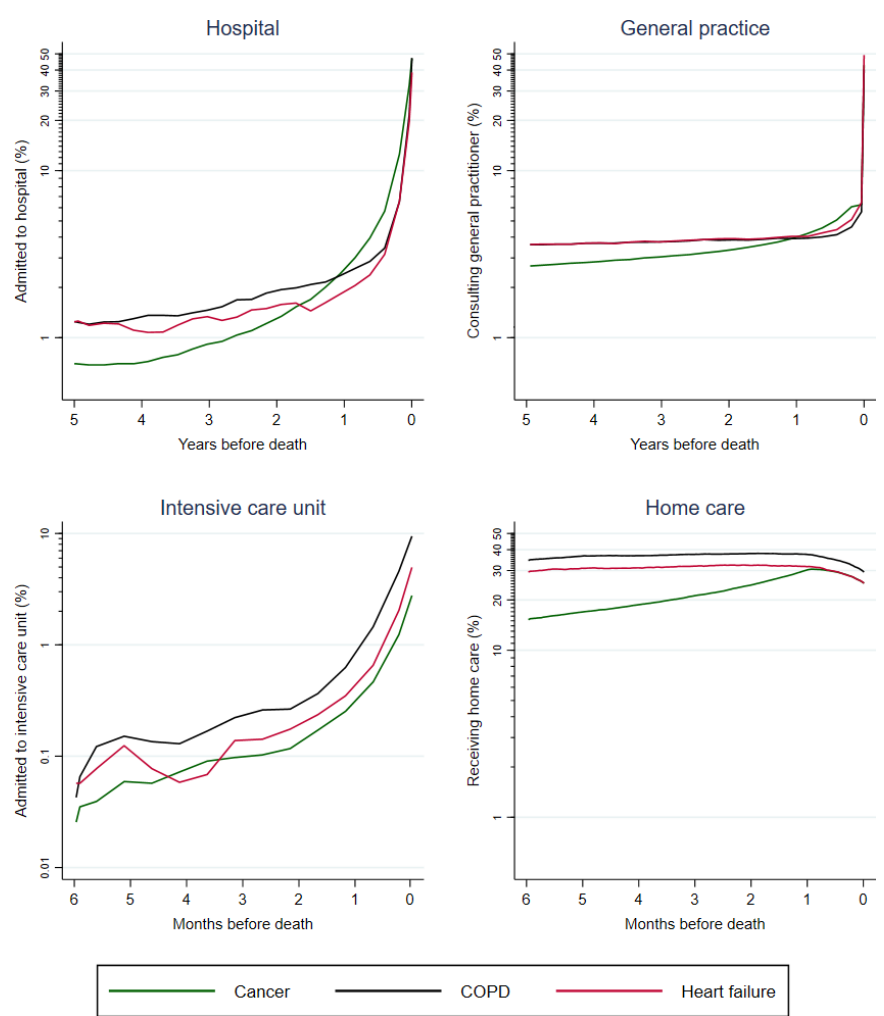

Figure 1 Daily prevalence proportions of patients admitted to hospital, admitted to intensive care, consulting a general practitioner or receiving home care according to underlying cause of death. COPD, chronic obstructive pulmonary disease.

\section{Consultations in general practice}

Within the last 5 years of life, $99.6 \%$ of the decedents consulted a general practitioner at least once.
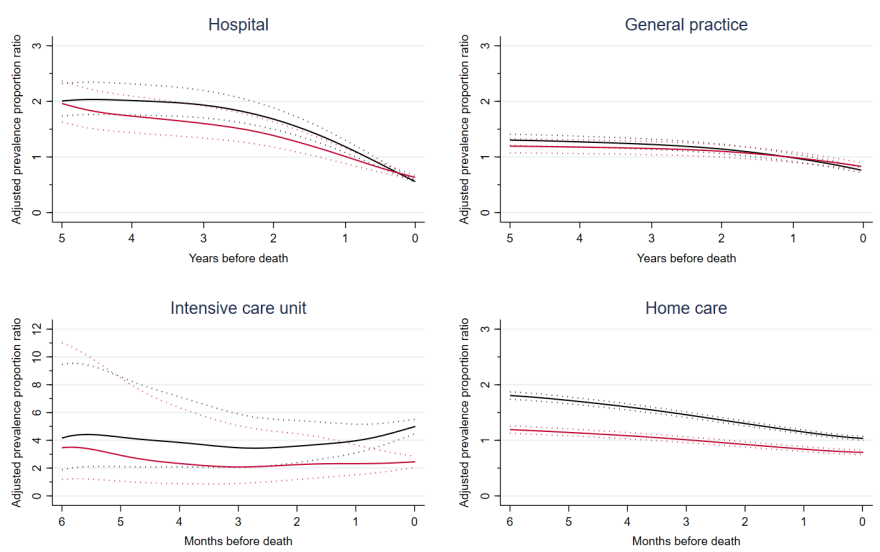

- COPD $\longrightarrow$ Heart fallure

Figure 2 Adjusted prevalence proportion ratios of patients with chronic obstructive pulmonary disease (COPD) or heart failure admitted to hospital, admitted to intensive care, consulting a general practitioner or receiving home care compared with patients with cancer.

A continuous increase in the daily PP of patients consulting a general practitioner was observed in all patient populations with a steep increase in the last months of life (figure 1). In this way, at 5 years before death, the daily $\mathrm{PP}$ of patients consulting a general practitioner was $3.5 \%$ (95\% CI $3.3 \%$ to $3.8 \%$ ) for patients with COPD, $3.9 \%$ (95\% CI $3.5 \%$ to $4.2 \%$ ) for patients with heart failure and $2.6 \%$ (95\% CI 2.5\% to 2.7\%) for patients with cancer. At day 30 before death, this was $5.1 \%(95 \%$ CI $4.8 \%$ to $5.4 \%$ ) for patients with COPD, $5.9 \%$ (95\% CI $5.4 \%$ to $6.3 \%$ ) for patients with heart failure and $6.5 \%$ $(95 \%$ CI $6.4 \%$ to $6.6 \%)$ for patients with cancer (table 2$)$.

Table 2 Daily prevalence proportion (PP) of patients admitted to hospital, admitted to intensive care, consulting a general practitioner or receiving home care according to underlying cause of death

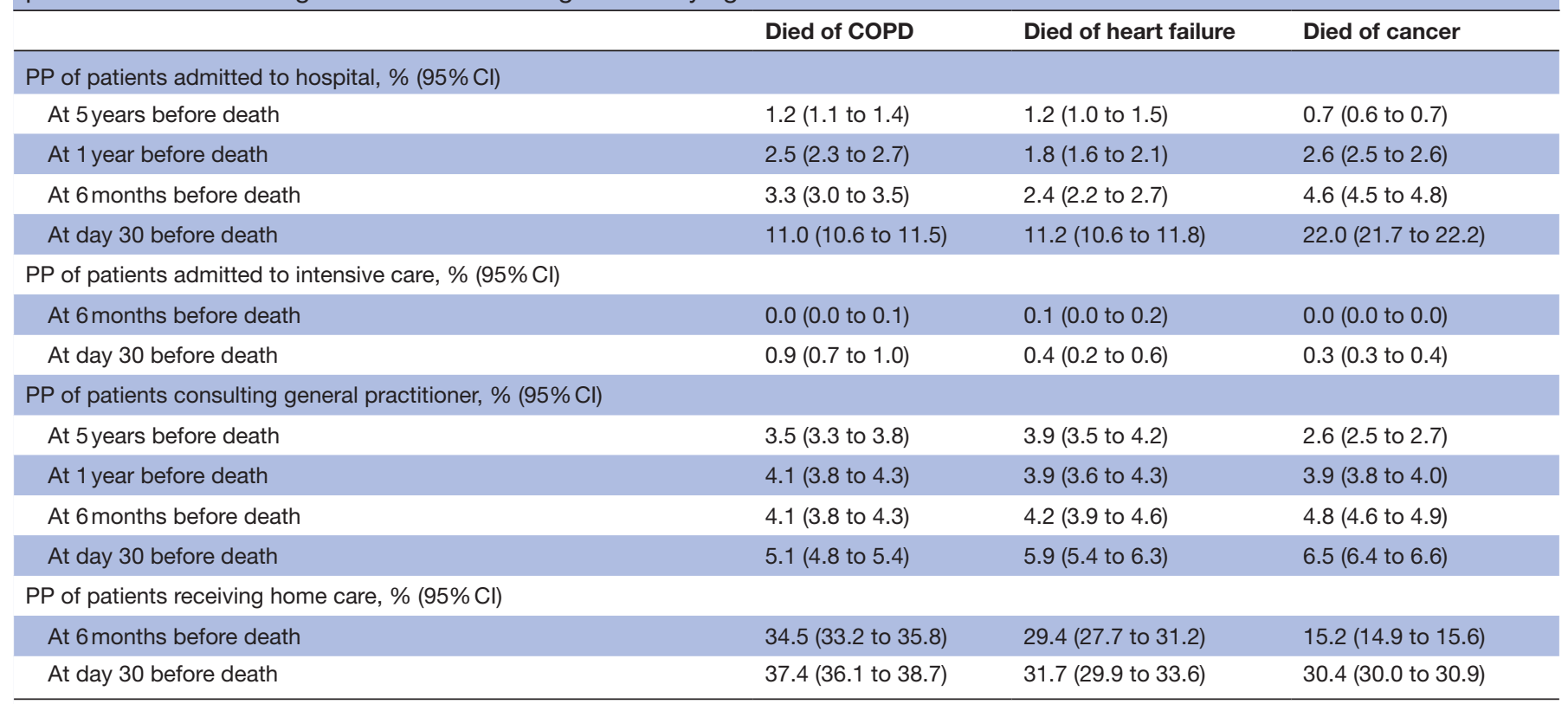

COPD, chronic obstructive pulmonary disease. 

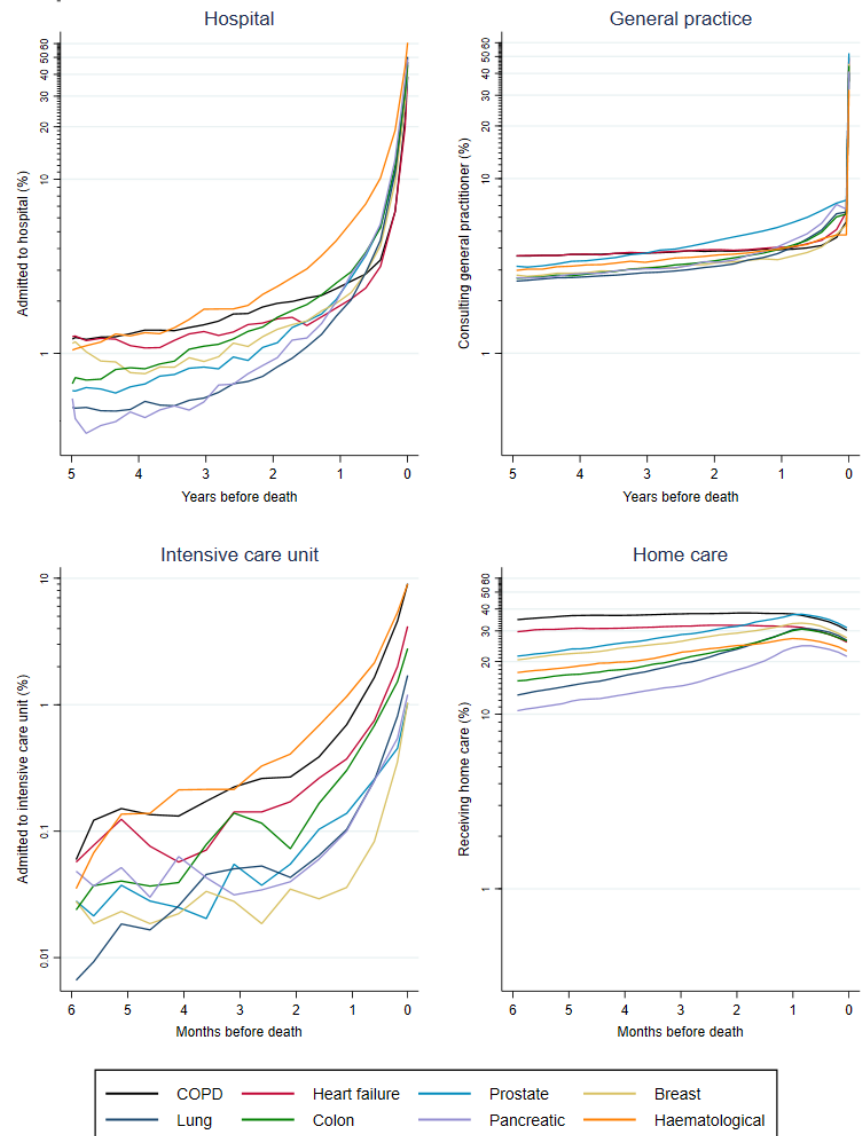

Figure 3 Daily prevalence proportions of patients admitted to hospital, admitted to intensive care, consulting a general practitioner or receiving home care according to specific type of cancer. COPD, chronic obstructive pulmonary disease.

The corresponding adjusted daily PPR for patients with COPD and heart failure when compared with patients with cancer remained unchanged until the final 2 years leading up to death in which it decreased slightly (figure 2).

Limited differences were found in course of daily PP between the non-cancer diseases, COPD and heart failure, and the specific types of cancer when stratifying on different types of cancer (figure 3).

\section{Intensive care unit admissions}

Within the last 6 months of life, $9.8 \%$ of the decedents were admitted to an intensive care unit at least once. Figure 1 shows low use of intensive care for all three patient populations and a modest increase during the last 6 months before death, accelerating in the last month. The daily PP at day 30 before death was $0.9 \%(95 \%$ CI $0.7 \%$ to $1.0 \%$ ) for patients with COPD, $0.4 \%$ (95\% CI $0.2 \%$ to $0.6 \%$ ) for patients with heart failure and $0.3 \%$ (95\% CI $0.3 \%$ to $0.4 \%$ ) for patients with cancer (table 2 ).

The adjusted daily PPR remained virtually unchanged for patients with COPD and heart failure when compared with patients with cancer during the 6 months period leading up to death (figure 2).
We found no differences when plotting daily PPs for patients with COPD and heart failure together with the specific types of cancer (figure 3 ).

\section{Home care provision}

Among the decedents, $40.6 \%$ received home care at least once within the last 6 months of life.

For patients dying from COPD or heart failure, the daily $\mathrm{PP}$ of receiving home care remained high but stable during the last 6 months of life with a slight decrease within the last month (figure 1). For patients dying from cancer, the daily PP was low but increased steadily during the last 6 months leading up to death with a decrease as for patients with COPD and heart failure within the last month of life (figure 1). Hence, the daily PP of patients receiving home care at 6 months before death was $34.5 \%$ (95\% CI $33.2 \%$ to $35.8 \%$ ) for patients with COPD, $29.4 \%$ (95\% CI $27.7 \%$ to $31.2 \%$ ) for patients with heart failure and $15.2 \%$ (95\% CI $14.9 \%$ to $15.6 \%$ ) for patients with cancer (table 2). Correspondingly, the daily PP at day 30 before death was $37.4 \%$ (95\% CI $36.1 \%$ to $38.7 \%$ ) for patients with COPD, $31.3 \%$ (95\% CI $29.9 \%$ to $33.6 \%$ ) for patients with heart failure and $30.4 \%$ (95\% CI $30.0 \%$ to $30.9 \%$ ) for patients with cancer (table 2).

The adjusted daily PPR for patients with COPD and heart failure when compared with patients with cancer decreased during the 6 months before death (figure 2).

When comparing the non-cancer diseases, COPD and heart failure, with the specific types of cancer, the trajectories of breast and prostate cancer mirrored the noncancer diseases the most with a more slowly increasing level of home care provision compared with the remaining types of cancer (figure 3 ).

\section{DISCUSSION}

We found limited differences in trajectories of healthcare resource utilisation at the end of life between people with COPD, heart failure or cancer when examining hospital admissions, including intensive care unit admissions, and consultations in general practice. For the provision of home care, patients with non-cancer received home care at a high but steady level in the months leading up to death, whereas patients with cancer were being increasingly cared for during the last 6 months of life.

However, when estimated by the use and timing of different healthcare resources, the results indicate comparable illness trajectories for the three patient populations.

Strengths of our study included its large-size nationwide population-based design and the possibility of accurate linkage between registries in the setting of a uniformly organised healthcare system. The national administrative and medical registries used in the study contain routinely and prospectively collected data. ${ }^{19} 2832$ There is some uncertainty about the classification of causes of death in the Danish Registry of Causes of Death, and, therefore, our analyses were based on three well-defined chronic illnesses to minimise misclassification. The Danish 
National Patient Registry and the National Health Insurance Service Registry are virtually complete registries in which only few admissions at hospital and consultations in general practice, respectively, are not registered. ${ }^{19} 32$ This indicates a low risk of information bias concerning patients' use of hospital and general practice at the end of life.

For the analyses on home care provision, we excluded patients in municipalities for which home care provision data had not been stated valid within the last 6 months leading up to death after ensuring comparability between included and excluded patients. ${ }^{29}$

The current study was based on the assumption that healthcare resource utilisation is a marker of illness progression and thereby functional impairment and symptom burden at the end of life. We tried to ascertain this by examining a wide range of types of care at the end of life. However, the remarkably steep increase in the daily PP of consultations in general practice on the day of death is not reflecting illness progression. This increase is rather due to the fact that on the day of death, decedents have been seen by a general practitioner who has made the declaration of death and written the death certificate.

Although analyses were adjusted for a wide range of potential confounders, we cannot exclude influence of residual confounding and confounding from unmeasured factors, on which information was not available in the current study, for example, socioeconomic status, severity of illness and lifestyle factors.

A concept of illness trajectories describing differences in the process of dying was originally developed by Glaser and Strauss in 1968. It includes four illness trajectories each differing in length and slope of physical decline, that is, 'organ failure', 'terminal illness', 'frailty' and 'sudden death'. ${ }^{11}$ The illness trajectory of organ failure, typical for those of patients with COPD or heart failure, has gradual physical decline over many years and episodes of acute deterioration before death due to exacerbations of their underlying disease with incomplete recovery. ${ }^{10}{ }^{11}$ The illness trajectory of terminal illness is considered most typical of patients with cancer with a steady physical decline and a clear declining terminal phase. ${ }^{1011}$

The current study suggests that the framework and the theoretical model of physical decline it has provided for end-of-life service planning and delivery may need to be reassessed. We did not discover the proposed distinctive trajectories when examining healthcare resource utilisation trajectories at the end of life. Only for the trajectories of home care provision, we found similarities with the model with patients with cancer having a clear increasing terminal provision of home care compared with patients with COPD and heart failure.

The interest of charting the course of the different illness trajectories leading up to death has been considerable in order to be able to tailor the best palliative care service prior to death for all patient populations and thereby diminish the substantial extent of potentially burdensome interventions at the end of life suggested in recent studies. ${ }^{33} 34$ Thus, several studies have tried to examine whether the concept of end-of-life illness trajectories can be ascertained in real-life data. ${ }^{35-41}$ Teno et al, Lunney et al and Stolz et al conducted studies measuring functional decline at the end of life, and all concluded that the underlying illness causing death was following the pattern of illness trajectories as suggested by Glaser and Strauss. ${ }^{11}{ }^{35-38}$ Contrary to this, in 2007, Gott et al examined illness trajectories of physical function experienced by patients with heart failure prior to death but discovered no typical heart failure trajectory for most of the patients included. ${ }^{39}$ In line with our study examining illness trajectories as reflected by healthcare resource utilisation, Sullivan et al set out to determine the pattern of medical expenses within 24 months before death among 1830 decedents dying from cancer, chronic disease or systemic failure. ${ }^{40}$ Similar to our findings on hospital admissions, intensive care unit admissions and consultations in general practice, though in a smaller study population, trajectories of medical expenses stayed relatively stable but increased in the last months of life for all patient populations. ${ }^{40}$ Correspondingly, Luta et al measured inpatient healthcare costs within 12 months before death among 108510 people dying from cancer, respiratory diseases or circulatory diseases and also found similar hospital cost trajectories for all patient populations. ${ }^{41}$ Except for Luta $e t$ al, previous studies all examined end-of-life illness trajectories in rather small study populations, whereas the current study aimed to ascertain illness trajectories in a larger nationwide populationbased setting.

Life-extending treatment has advanced tremendously for several types of cancer over the past decade, and many more patients are now living with metastatic disease, for example, breast and prostate cancer. This may also explain the different findings in our study compared with some of the previous studies, since these treatment improvements have reshaped the illness trajectories which, for some cancer types, may now mirror that of non-cancer diseases with a slowly declining physical function over many years and episodes of acute deterioration before death. ${ }^{42}{ }^{43}$ In this way, end-of-life healthcare services for patients with cancer may have evolved towards being offered according to patients' needs rather than prognosis. ${ }^{44}$ This is in line with the findings in the current study, in which we experienced the home care provision trajectories for breast and prostate cancer to differ from the remaining cancer types and be more like the trajectories of COPD and heart failure.

The current study is not able to directly determine potential differences in needs of care at the end of life among patients with different chronic diseases. Hence, we cannot conclude whether the observed differences in endof-life healthcare resource utilisation between patients without cancer and with cancer reflect inequalities in provision of services or differences in need. However, a number of studies highlight the equivalent or even greater symptom burden in non-cancer diseases compared with 
cancer, causing symptom management inequalities for equally needy patient populations. ${ }^{45-49}$ Thus, it emphasises that palliative care interventions should be triggered by severity of symptoms rather than underlying disease, prognosis and treatment intention. ${ }^{50}{ }^{51}$ Patients do not have to forego curative treatment or be expected to die within short time to receive palliative treatment, that is, palliative care interventions can be established alongside life-prolonging or curative treatments in order to prevent rather than simply relieve suffering. ${ }^{52}$

In addition, it has been suggested that palliative care for non-cancer diseases improves quality of life, reduce costly and potentially burdensome hospital admissions and increase the chance of dying at home. ${ }^{1}$

We believe the quite parallel healthcare utilisation trajectories at the end of life for patients without cancer and with cancer, respectively, suggest comparable progression in illness severity and functional impairment at the end of life, and this warrants consideration. Hence, current efforts, based on the concept of illness trajectories ${ }^{11}$ to plan and deliver palliative care services, both generalist and specialist, for people with non-cancer diseases may need to be reconsidered in order to allocate resources more adequately. Moreover, developing palliative care services in non-cancer diseases must remain a priority.

\section{CONCLUSION}

The healthcare resource utilisation trajectories at the end of life for people with COPD or heart failure differ little from those with cancer. When estimated by the use of hospital, including intensive care and general practitioner, the illness trajectories at the end of life were found to be comparable for the three patient populations. For the provision of home care, the trajectories of breast and prostate cancer, mirrored that of patients with COPD and heart failure. This emphasises the fact that provision of palliative care must be based on palliative needs rather than diagnosis in order to ensure access to adequate care for people with non-cancer as well as cancer diagnoses.

\section{Author affiliations \\ ${ }^{1}$ Department of Clinical Epidemiology, Aarhus University Hospital, Aarhus N, Denmark \\ ${ }^{2}$ Department of Clinical Medicine, Aarhus University, Aarhus C, Denmark \\ ${ }^{3}$ The Palliative Care Team, Department of Oncology, Aarhus University Hospital, Aarhus N, Denmark \\ ${ }^{4}$ Danish Center for Clinical Health Services Research, Aalborg University Hospital, Aalborg $\emptyset$, Denmark \\ ${ }^{5}$ Department of Clinical Medicine, Aalborg University, Aalborg $\emptyset$, Denmark}

Contributors AHSV, MAN, CFC and SPJ initiated and designed the study. Acquisition of data was done by AHSV, CFC and SPJ. AHSV performed the statistical analysis with assistance from JBV. AHSV drafted the manuscript which was critically reviewed by all authors. All read and approved the final version of the manuscript. AHSV is acting as guarantor.

Funding This works was supported by the Independent Research Fund Denmark, grant number: 4004-00609B; Fonden af 1870, grant number: 200652; C.C. Klestrup \& Hustru Henriette Klestrups Mindelegat, grant number: 10761; Fabrikant Einar Willumsens Mindelegat, grant number: 131217.

Competing interests None declared.
Patient and public involvement Patients and/or the public were not involved in the design, or conduct, or reporting, or dissemination plans of this research.

Patient consent for publication Not applicable.

Ethics approval In accordance with Danish law, non-interventional studies do not require approval from ethics committees. The study was reported to the Danish Data Protection Agency (record number 2015-57-0002) by registration at Aarhus University (Aarhus University record number 2016-051-000001/977).

Provenance and peer review Not commissioned; externally peer reviewed.

Data availability statement Data are available upon reasonable request. No data are available. All data relevant to the study are included in the article or uploaded as supplementary information.Data are only available as presented in the paper. According to Danish legislation, our approvals to use the Danish data sources for the current study do not allow us to distribute or make patient data directly available to other parties.

Open access This is an open access article distributed in accordance with the Creative Commons Attribution Non Commercial (CC BY-NC 4.0) license, which permits others to distribute, remix, adapt, build upon this work non-commercially, and license their derivative works on different terms, provided the original work is properly cited, appropriate credit is given, any changes made indicated, and the use is non-commercial. See: http://creativecommons.org/licenses/by-nc/4.0/.

\section{ORCID iDs}

Anne Høy Seemann Vestergaard http://orcid.org/0000-0002-5140-1803

Christian Fynbo Christiansen http://orcid.org/0000-0002-0727-953X

Jan Brink Valentin http://orcid.org/0000-0002-8205-7179

\section{REFERENCES}

1 Quinn KL, Stukel T, Stall NM, et al. Association between palliative care and healthcare outcomes among adults with terminal noncancer illness: population based matched cohort study. BMJ 2020;370:m2257.

2 Brumley RD, Enguidanos S, Cherin DA. Effectiveness of a homebased palliative care program for end-of-life. J Palliat Med 2003;6:715-24

3 Veronese S, Gallo G, Valle A, et al. Specialist palliative care improves the quality of life in advanced neurodegenerative disorders: NE-PAL, a pilot randomised controlled study. BMJ Support Palliat Care 2017;7:164-72.

4 Aiken LS, Butner J, Lockhart CA, et al. Outcome evaluation of a randomized trial of the PhoenixCare intervention: program of case management and coordinated care for the seriously chronically ill. $J$ Palliat Med 2006;9:111-26.

5 Sidebottom AC, Jorgenson A, Richards $\mathrm{H}$, et al. Inpatient palliative care for patients with acute heart failure: outcomes from a randomized trial. J Palliat Med 2015;18:134-42.

6 Rogers JG, Patel CB, Mentz RJ, et al. Palliative care in heart failure: the PAL-HF randomized, controlled clinical trial. J Am Coll Cardiol 2017;70:331-41.

7 Wong FKY, Ng AYM, Lee PH, et al. Effects of a transitional palliative care model on patients with end-stage heart failure: a randomised controlled trial. Heart 2016;102:1100-8.

8 Knaul FM, Farmer PE, Krakauer EL, et al. Alleviating the access abyss in palliative care and pain relief-an imperative of universal health coverage: the Lancet Commission report. Lancet 2018;391:1391-454.

9 Bloom Cl, Slaich B, Morales DR, et al. Low uptake of palliative care for COPD patients within primary care in the UK. Eur Respir J 2018;51. doi:10.1183/13993003.01879-2017. [Epub ahead of print: 1402 2018].

10 Murray SA, Kendall M, Boyd K, et al. Illness trajectories and palliative care. BMJ 2005;330:1007-11.

11 Glaser BG, Strauss AL. Time for dying. Chicago, IL: Aldine Publishing, 1968: 68. 2660.

12 Gill TM, Gahbauer EA, Han L, et al. The role of intervening hospital admissions on trajectories of disability in the last year of life: prospective cohort study of older people. BMJ 2015;350:h2361.

13 Pinnock $\mathrm{H}$, Kendall M, Murray SA, et al. Living and dying with severe chronic obstructive pulmonary disease: multi-perspective longitudinal qualitative study. BMJ 2011;342:d142.

14 Oishi A, Murtagh FEM. The challenges of uncertainty and interprofessional collaboration in palliative care for non-cancer patients in the community: a systematic review of views from 
patients, carers and health-care professionals. Palliat Med 2014;28:1081-98.

15 Mainz J, Hess MH, Johnsen SP. The Danish unique personal identifier and the Danish civil registration system as a tool for research and quality improvement. Int J Qual Health Care 2019;31:717-20.

16 Schmidt M, Schmidt SAJ, Adelborg K, et al. The Danish health care system and epidemiological research: from health care contacts to database records. Clin Epidemiol 2019;11:563-91.

17 Danish national register of causes of D. year report 20122012.

18 Helweg-Larsen K. The Danish register of causes of death. Scand J Public Health 2011;39:26-9.

19 Schmidt M, Schmidt SAJ, Sandegaard JL, et al. The Danish national patient registry: a review of content, data quality, and research potential. Clin Epidemiol 2015;7:449-90.

20 Charlson ME, Pompei P, Ales KL, et al. A new method of classifying prognostic comorbidity in longitudinal studies: development and validation. J Chronic Dis 1987;40:373-83.

21 Thygesen SK, Christiansen CF, Christensen S, et al. The predictive value of ICD-10 diagnostic coding used to assess Charlson comorbidity index conditions in the population-based Danish national Registry of patients. BMC Med Res Methodol 2011;11:83-2288-11-83.

22 Schmidt M, Pedersen L, Sørensen HT. The Danish civil registration system as a tool in epidemiology. Eur J Epidemiol 2014;29:541-9.

23 Jensen VM, Rasmussen AW. Danish education registers. Scand J Public Health 2011;39:91-4.

24 Baadsgaard M, Quitzau J. Danish registers on personal income and transfer payments. Scand J Public Health 2011;39:103-5.

25 Statistics Denmark. INDKF201: main table for family income statistics by type of income, family type, population, price unit and unit 20042019. Available: www.statistikbanken.dk/INDKF201 [Accessed 22 Jun 2021].

26 Andersen JS, Olivarius NDF, Krasnik A. The Danish National health service register. Scand J Public Health 2011;39:34-7.

27 Christiansen CF, Møller MH, Nielsen $\mathrm{H}$, et al. The Danish intensive care database. Clin Epidemiol 2016;8:525-30.

28 Christiansen CF, Fau CS, Johansen MB, et al. The impact of preadmission morbidity level on 3-year mortality after intensive care: a Danish cohort study. (1399-6576 (electronic)).

29 Statistics Denmark. Home care Provison, months of reporting by municipalities, 2018.

30 Statistics Denmark. Elderly indicators 2021. Available: https://www. dst.dk/en/Statistik/dokumentation/documentationofstatistics/elderlyindicators [Accessed 22 Jun 2021].

31 Sundhedsdatastyrelsen. Danish registry of causes of death. year report 2016, 2017.

32 Olivarius NF, Hollnagel H, Krasnik A, et al. The Danish National health service register. A tool for primary health care research. Dan Med Bull 1997:44:449-53.

33 Mason B, Kerssens JJ, Stoddart A, et al. Unscheduled and out-ofhours care for people in their last year of life: a retrospective cohort analysis of national datasets. BMJ Open 2020;10:e041888.

34 Vestergaard AHS, Neergaard MA, Christiansen CF, et al. Hospitalisation at the end of life among cancer and non-cancer patients in Denmark: a nationwide register-based cohort study. BMJ Open 2020;10:e033493.

35 Teno JM, Weitzen S, Fennell ML, et al. Dying trajectory in the last year of life: does cancer trajectory fit other diseases? J Palliat Med 2001;4:457-64.

36 Lunney JR, Lynn J, Foley DJ, et al. Patterns of functional decline at the end of life. JAMA 2003;289:2387-92.

37 Lunney JR, Albert SM, Boudreau R, et al. Mobility trajectories at the end of life: comparing clinical condition and latent class approaches. J Am Geriatr Soc 2018;66:503-8.

38 Stolz E, Gill TM, Mayerl H. Trajectories of late-life disability vary by the condition leading to death. J Gerontol A Biol Sci Med Sci 2020.

39 Gott M, Barnes S, Parker C, et al. Dying trajectories in heart failure. Palliat Med 2007;21:95-9.

40 Sullivan SS, Li J, Wu Y-WB, et al. Complexity of chronic conditions' impact on end-of-life expense trajectories of Medicare decedents. $J$ Nurs Adm 2017;47:545-50.

41 Luta X, Diernberger K, Bowden J, et al. Healthcare trajectories and costs in the last year of life: a retrospective primary care and hospital analysis. BMJ Support Palliat Care 2020. doi:10.1136/ bmjspcare-2020-002630. [Epub ahead of print: 02 Dec 2020].

42 Alwan A. Global status report on noncommunicable diseases 2010. World Health Organization, 2011.

43 Reed E, Corner J. Defining the illness trajectory of metastatic breast cancer. BMJ Support Palliat Care 2015;5:358-65.

44 Kaasa S, Loge JH, Aapro M, et al. Integration of oncology and palliative care: a Lancet oncology Commission. Lancet Oncol 2018;19:e588-653.

45 See D, Le B, Gorelik A, et al. Symptom burden in malignant and non-malignant disease on admission to a palliative care unit. $B M J$ Support Palliat Care 2019. doi:10.1136/bmjspcare-2018-001560. [Epub ahead of print: 04 Feb 2019].

46 De Korte-Verhoef MC, Pasman HRW, Schweitzer BPM, et al. Reasons for hospitalisation at the end of life: differences between cancer and non-cancer patients. Support Care Cancer 2014;22:645-52.

47 Moens K, Higginson IJ, Harding R, et al. Are there differences in the prevalence of palliative care-related problems in people living with advanced cancer and eight non-cancer conditions? A systematic review. J Pain Symptom Manage 2014;48:660-77.

48 Bekelman DB, Rumsfeld JS, Havranek EP, et al. Symptom burden, depression, and spiritual well-being: a comparison of heart failure and advanced cancer patients. J Gen Intern Med 2009;24:592-8.

49 Ostgathe C, Alt-Epping B, Golla H, et al. Non-Cancer patients in specialized palliative care in Germany: what are the problems? Palliat Med 2011;25:148-52.

50 Rome RB, Luminais $\mathrm{HH}$, Bourgeois DA, et al. The role of palliative care at the end of life. Ochsner J 2011;11:348-52.

51 Kelley AS, Morrison RS. Palliative care for the seriously ill. N Engl J Med 2015;373:747-55.

52 World Health Organization. Who definition of palliative care. Available: https://www.who.int/cancer/palliative/definition/en/2021 [Accessed 30 Jun 2021]. 